\title{
Early-onset type 2 diabetes in obese white subjects is characterised by a marked defect in beta cell insulin secretion, severe insulin resistance and a lack of response to aerobic exercise training
}

\author{
N. Burns • F. M. Finucane $\cdot$ M. Hatunic • \\ M. Gilman • M. Murphy • D. Gasparro • A. Mari • \\ A. Gastaldelli • J. J. Nolan
}

Received: 12 January 2007 / Accepted: 23 February 2007 / Published online: 29 March 2007

(C) Springer-Verlag 2007

\begin{abstract}
Aims/hypothesis Early-onset type 2 diabetes is associated with marked visceral obesity and extreme insulin resistance, but its pathogenesis and response to treatment are not completely understood. We studied physical fitness, wholebody and hepatic glucose turnover, and insulin secretion in young obese Irish subjects before and after 3 months of aerobic exercise training. We hypothesised that exercise alone, with stable diet, should improve insulin sensitivity.

Materials and methods Anthropometric parameters and maximum volume of oxygen utilisation $\left(V_{2} \mathrm{O}_{2 \max }\right)$ were measured in 13 subjects with type 2 diabetes and 18 nondiabetic control subjects, matched for age and BMI. Insulin sensitivity and hepatic glucose turnover were measured using the hyperinsulinaemic-euglycaemic clamp. Insulin secretion was assessed from an OGTT and a modified
\end{abstract}

N. Burns and F. M. Finucane contributed equally to this work.

N. Burns $\cdot$ F. M. Finucane $\cdot$ M. Hatunic $\cdot$ M. Gilman •

M. Murphy $\cdot$ J. J. Nolan $(\bowtie)$

Metabolic Research Unit, Department of Endocrinology,

Hospital 5, St James' Hospital, Trinity College Dublin,

Dublin 8, Ireland

e-mail: jnolan@stjames.ie

D. Gasparro

Department of Chemical Pathology, St James' Hospital,

Dublin, Ireland

\section{A. Mari}

Institute of Biomedical Engineering, National Research Council, Padua, Italy

A. Gastaldelli

CNR Institute,

Pisa, Italy intravenous glucose tolerance test. Some subjects (seven type 2 diabetic, 14 non-diabetic control subjects) then completed a 12-week supervised aerobic exercise programme. All measurements were repeated on completion of the exercise programme.

Results Type 2 diabetic subjects had higher WHR, systolic blood pressure and triacylglycerols than non-diabetic control subjects. They were significantly more insulinresistant as measured both by the clamp and oral glucose insulin sensitivity. They also displayed marked defects in insulin secretion in response to oral and intravenous glucose challenges. Exercise intervention had no significant effect on whole-body or hepatic insulin sensitivity or insulin secretion. $V \mathrm{O}_{2 \max }$ increased significantly in the non-diabetic control subjects, but not in the type 2 diabetic subjects after exercise training.

Conclusions/interpretation Young obese subjects with type 2 diabetes are severely insulin-resistant with marked loss of beta cell function compared with control subjects matched for age and obesity. Neither group responded metabolically to aerobic exercise intervention.

Keywords Early onset - Beta cell function · Exercise . Glucose sensitivity · Hyperinsulinaemic clamp . Insulin resistance $\cdot$ Insulin secretion · Type 2 diabetes · Visceral Obesity. Young
Abbreviations
GLUT4 glucose transporter 4
IVGTT intravenous glucose tolerance test
OGIS oral glucose insulin sensitivity
$\mathrm{VO}_{2 \max }$ maximum volume of oxygen utilisation 


\section{Introduction}

Marked visceral obesity and extreme insulin resistance are associated with early-onset type 2 diabetes. The prevalence of diabetes is predicted to more than double to 366 million by 2030 [1]. A more recent trend has been the shift of type 2 diabetes to middle-aged and younger populations. The new epidemic of obesity and type 2 diabetes in young people is becoming a clinical and health economic priority [2].

In Ireland, in the last decade, the prevalence of obesity has increased by $67 \%$ [3]. Irish adolescent boys and girls ranked third and second highest respectively in an international study of obesity prevalence rates [4]. This has been associated with significant changes in physical activity and diet in this age group [5]. However, the exact sequence of events leading to type 2 diabetes in youth is not fully understood. We have reported that younger Irish adults (aged less than 40 years) with type 2 diabetes are more obese, more dyslipidaemic and have worse initial and ongoing glycaemic control than older adults with type 2 diabetes in spite of similar treatment protocols [6]. In a separate study of younger white Irish subjects with type 2 diabetes (mean age 22 years), we used an insulin-modified intravenous glucose tolerance test (IVGTT) to demonstrate severe insulin resistance and loss of first-phase insulin secretion [7]. This cohort had evidence of high risk for cardiovascular complications, with elevated markers of endothelial dysfunction. This suggests that a disproportionate cardiovascular risk burden is associated with type 2 diabetes in younger patients. To date there have been no studies of insulin sensitivity using the hyperinsulinaemiceuglycaemic clamp technique in this population.

There is considerable evidence that exercise delays or prevents the development of type 2 diabetes in at-risk populations [8-12]. Smaller studies have shown that exercise improves insulin sensitivity and glucose metabolism $[13,14]$. We have recently shown that short-term exercise training increased insulin sensitivity by more than $50 \%$ in obese middle-aged patients (mean age 45 years) with type 2 diabetes [15]. In these patients the main biochemical change was an increase in glucose transporter 4 (GLUT4) protein content in skeletal muscle. These improvements, while impressive, are likely to be proportional to the duration and intensity of the exercise regime [16]. The metabolic benefits of exercise are known to be short-lived [17-20]. This has important implications for the design and optimisation of exercise interventions.

The aim of the current study was to examine the effects of a 3-month aerobic exercise training programme in young obese insulin-resistant subjects with and without type 2 diabetes. We hypothesised that exercise would lead to an improvement in insulin sensitivity in these severely insulinresistant subjects. We used the glucose clamp and tracer techniques to measure the effects of exercise on wholebody and hepatic insulin sensitivity and insulin secretion.

\section{Subjects and methods}

Study population

Patients aged between 15 and 30 years, who were attending either the endocrinology or diabetes services at St James' Hospital, Dublin and had obesity or type 2 diabetes, were invited to take part in the study. Subjects with co-existing illnesses or secondary forms of diabetes were excluded. The study protocol was approved by the local Research Ethics Committee and written informed consent was obtained.

\section{Screening}

Subjects attended the Metabolic Research Unit on one morning at $08.00 \mathrm{~h}$ for documentation of their full history and a physical examination. Routine blood samples were taken for blood count, renal, liver, bone profile, thyroid function tests and fasting lipids. Each subject with diabetes was confirmed negative for GAD antibodies.

\section{Anthropometric measurements}

Waist-to-hip ratio, weight, height and BMI were measured. Blood pressure was measured using the left arm after the subject had been sitting comfortably for $5 \mathrm{~min}$, using an oscillometric device (Omron 705 CP; Omron, Matsusaka, Japan). Three readings were taken and the lowest one recorded. Body composition was assessed using an electrical impedance device (Tanita TBF-300 Body Composition Analyser; Tanita, Tokyo, Japan). Urinalysis and 12-lead ECG were performed.

Testing of maximum volume of oxygen utilisation

Maximum volume of oxygen utilisation $\left(\mathrm{VO}_{2 \max }\right)$ was measured using a bicycle ergometer (Excalibur, Groningen, the Netherlands) in a stepwise fashion. Heart rate and oxygen consumption were recorded. Blood pressure was monitored.

\section{OGTT}

An OGTT (75 g glucose load) was performed. Insulin and $\mathrm{C}$ - peptide levels were measured in addition to blood glucose, at 30-min intervals over $2 \mathrm{~h}$.

Hyperinsulinaemic-euglycaemic clamp study

On a separate morning (within 7 days of the OGTT), subjects attended the unit for a 4-h hyperinsulinaemic- 
euglycaemic clamp study. Subjects fasted for $12 \mathrm{~h}$ prior to the clamp. Diabetic subjects taking insulin omitted the basal dose on the night before the clamp as well as the dose on the morning of the clamp study. Subjects taking metformin stopped this 1 week before the study, while subjects taking sulfonylureas stopped these 3 days before the clamp. During a 2 -h basal phase, $\left[6,6-{ }^{2} \mathrm{H}_{2}\right]$ glucose tracer (Cambridge Isotopes, Cambridge, MA, USA) was infused through an intravenous cannula in the right antecubital fossa. Subsequently a 2-h insulin infusion (Actrapid; Novo Nordisk, Bagsvaerd, Denmark) was commenced through an intravenous cannula in the left antecubital fossa at a rate of $40 \mathrm{mU} \mathrm{m}^{-2} \mathrm{~min}^{-1}$. Simultaneously a $\left[6,6-{ }^{2} \mathrm{H}_{2}\right]$ glucose solution was infused in order to maintain euglycaemia. To monitor blood glucose levels during the clamp study, arterialised venous blood was taken at 5-min intervals from a retrograde cannula on the dorsum of the right hand, which was warmed. The mean glucose infusion rate between 80 and $120 \mathrm{~min}$ after commencement of the insulin infusion was calculated. The glucose disposal rate was calculated after correction for residual hepatic glucose production using the $\left[6,6-{ }^{2} \mathrm{H}_{2}\right]$ glucose tracer $[21-23]$.

\section{Modified intravenous glucose tolerance test}

At the end of the clamp study (at $240 \mathrm{~min}$ ), a rapid bolus of intravenous glucose was administered $(0.3 \mathrm{~g} / \mathrm{kg})$ over $20 \mathrm{~s}$ and serial blood samples taken every $2 \mathrm{~min}$ for $8 \mathrm{~min}$ to measure insulin, C-peptide and glucose. The IVGTT has been used in similar cohorts to assess beta cell function in the past [7].

\section{Analysis methods for OGTT and IVGTT data}

Beta cell function was assessed using a model that describes the relationship between insulin secretion and glucose concentration and that has been illustrated in detail previously $[24,25]$. The model expresses insulin secretion as a sum of two components. The first component represents the dependence of insulin secretion on absolute glucose concentration at any time point and is characterised by a dose-response function relating the two variables. The characteristic parameter of the dose-response is the mean slope within the observed glucose range, denoted as 'beta cell glucose sensitivity.' The dose-response is modulated by a potentiation factor, which accounts for several potentiating factors (prolonged exposure to hyperglycaemia, non-glucose substrates, gastrointestinal hormones and neurotransmitters). The potentiation factor is set to be a positive function of time and to average 1 during the experiment. It thus expresses a relative potentiation of the secretory response to glucose. Previous studies [24-26] have found that insulin secretion at the end of an OGTT or meal is relatively higher than at the beginning for comparable glucose levels (i.e. when glucose returns to the basal level, insulin secretion remains higher). Potentiation thus increases during the test. This increase has been quantified as the ratio between the potentiation factor value at $2 \mathrm{~h}$ after the OGTT and that at time zero. The second insulin secretion component represents a dynamic dependence of insulin secretion on the rate of change of glucose concentration. This component is termed the derivative component and is determined by a single parameter, denoted as 'rate sensitivity.' Rate sensitivity is related to early insulin release [24-26].

The model parameters were estimated from glucose and C-peptide concentrations by regularised least-squares, as previously described $[24,25]$. Regularisation involves the choice of smoothing factors that were selected to obtain glucose and C-peptide model residuals with standard deviations close to the expected measurement error $(\sim 1 \%$ for glucose and $\sim 4 \%$ for C-peptide). Basal and total insulin secretion during the OGTT were calculated from the estimated model parameters. Total insulin secretion was calculated as the integral of insulin secretion during the OGTT. Insulin secretion was expressed in pmol $\mathrm{min}^{-1} \mathrm{~m}^{-2}$ of body surface area.

The acute insulin response was calculated as the mean incremental insulin concentration during the 8-min IVGTT, where the mean was determined by trapezoidal integration. Analogous indices were obtained using C-peptide concentration and insulin secretion, which was calculated by deconvolution from C-peptide concentration [27].

\section{Laboratory analysis}

Serum insulin and C-peptide were measured using commercially available flouroimmunoassays (Auto-Delfia, WallacOy, Finland). Plasma total cholesterol and triacylglycerols were measured using enzymatic methods (Human liquicolor kits; Hitachi Modular; Roche Diagnostics, Basel Switzerland). Plasma HDL-cholesterol and LDL-cholesterol were measured directly with enzymatic methods (Randox direct kits; Hitachi Modular). Plasma glucose was measured using a glucose oxidase method (bio Merieux kit; Hitachi Modular) and $\mathrm{HbA}_{1 \mathrm{c}}$ was measured using an analyser (HiAuto A1c HA 8140; Menarini, Florence Italy). Antibodies to GAD were measured using a direct radioligand assay. Serum NEFA were determined using a spectrophotometric assay (Randox Laboratories, Antrim, UK).

\section{Exercise protocol}

The exercise programme was conducted entirely at the Metabolic Research Unit gym and involved $1 \mathrm{~h}$ of exercise training, four times per week for 12 weeks. Each exercise session was supervised by an exercise physiologist or one of the study physicians. Each subject completed a 5-min 
warm-up, followed by $60 \mathrm{~min}$ of aerobic exercise at $70 \%$ $V \mathrm{O}_{2 \text { max }}$ (with continuous heart rate monitoring) and a 5-min cool down either on a cycle ergometer or treadmill. Compliance with the exercise was excellent with $95 \%$ attendance throughout the study.

Diet

A dietitian met with each subject weekly to ensure a stable energy intake during the study.

Statistical analysis

Data are presented as means (SEM). Comparisons between control and young type 2 diabetes subjects at baseline (before exercise) used a Mann-Whitney $U$ test. Comparisons of both of these groups before and after exercise used the Wilcoxon signed rank test. Statistical significance was set at $p<0.05$. JMP statistical software (version 5.1; SAS Institute, Cary, NC, USA) was used in statistical analysis.

\section{Results}

Baseline studies

Clinical and laboratory measurements We recruited 13 subjects with type 2 diabetes and 18 non-diabetic control subjects for the baseline study. Clinical characteristics of the subjects are shown in Table 1. The two groups were matched for age, BMI, body fat and physical fitness. In seven of the 13 subjects with type 2 diabetes and 11 of the 18 obese subjects there was a documented family history of type 2 diabetes. All of the subjects had completed puberty. Of the 13 type 2 diabetic subjects, six were receiving metformin alone, two were receiving metformin with sulfonylureas, three were receiving insulin (alone or in combination with oral agents) and two were on no hypoglycaemic medications. No differences were subsequently noted in the responses between these treatment sub-groups. There was a higher proportion of men in the type 2 diabetic subjects group, which accounted for a non-significant difference in weight, height, body surface area and fat-free mass. None of these differences were significant when the sexes were analysed separately (not shown). The type 2 diabetic subjects had higher waist circumference, WHR, fasting triacylglycerol and slightly higher systolic blood pressure than the obese control subjects. In the subgroup (14 obese control, seven type 2 diabetic subjects) who completed the exercise intervention, the baseline trends were similar.

Hyperinsulinaemic-euglycaemic clamp studies Basal hepatic glucose output was increased in the type 2 diabetic subjects $\left(2.6 \pm 0.25 \mathrm{mg} \mathrm{kg} \mathrm{min}^{-1}\right)$ compared with the obese control subjects $\left(1.72 \pm 0.14 \mathrm{mg} \mathrm{kg}{ }^{-1} \min ^{-1}\right.$; $p=0.0015$ ). Glucose production was suppressed to a similar
Table 1 Clinical data, lipid profiles and metabolic data derived from the OGTT prior to exercise intervention: comparison between obese control and obese type 2 diabetic subjects

Values in brackets: SEM

${ }^{\mathrm{a}} \mathrm{pmol} \mathrm{min}^{-1} \mathrm{~m}^{-2} \mathrm{mmol}^{-1} \mathrm{l}^{-1}$

b $\mathrm{pmol} \mathrm{m}^{-2} \mathrm{mmol}^{-1} \mathrm{l}^{-1}$

c $\mathrm{pmol} \mathrm{min}^{-1} \mathrm{~m}^{-2}$

d $\mathrm{nmol} / \mathrm{m}^{2}$

$* p<0.05 ; * * p<0.01$;

$* * * p<0.001$

\begin{tabular}{|c|c|c|}
\hline & Control $(n=18)$ & Type 2 diabetic subjects $(n=13)$ \\
\hline Male:female ratio & $4: 14$ & $8: 5$ \\
\hline Mean age (years) & $23.7(0.9)$ & $25.8(1.2)$ \\
\hline Weight $(\mathrm{kg})$ & $99.6(4.4)$ & $109(5)$ \\
\hline BMI $\left(\mathrm{kg} / \mathrm{m}^{2}\right)$ & $35.2(1.4)$ & $34.9(1.4)$ \\
\hline \multicolumn{3}{|l|}{ Blood pressure (mmHg) } \\
\hline Systolic & $114.9(3.3)$ & $124.9(3.4) *$ \\
\hline Diastolic & $72.9(2.1)$ & $77.5(2.5)$ \\
\hline Waist circumference $(\mathrm{cm})$ & $102.1(2.72)$ & $117.2(3.8) * *$ \\
\hline Hip circumference $(\mathrm{cm})$ & $116.4(3.3)$ & $115.3(2.8)$ \\
\hline WHR & $0.88(0.02)$ & $1.02(0.03) * *$ \\
\hline Body fat $\%$ & $41.2(2.15)$ & $38.3(3.02)$ \\
\hline$V \mathrm{O}_{2 \max }\left(\mathrm{ml} \mathrm{min}^{-1} \mathrm{~kg}^{-1}\right)$ & $2.66(0.19)$ & $2.55(0.2)$ \\
\hline Fasting plasma glucose $(\mathrm{mmol} / \mathrm{l})$ & $4.94(0.34)$ & $10.31(0.42) * * *$ \\
\hline Total cholesterol (mmol/l) & $4.42(0.2)$ & $4.69(0.24)$ \\
\hline HDL-cholesterol (mmol/l) & $1.09(0.07)$ & $0.94(0.04)$ \\
\hline LDL-cholesterol (mmol/l) & $2.44(0.17)$ & $2.67(0.2)$ \\
\hline Triacylglycerol (mmol/l) & $1.49(0.19)$ & $2.9(0.37) * * *$ \\
\hline Fasting plasma NEFA (mmol/l) & $0.642(0.041)$ & $0.748(0.236)$ \\
\hline $\mathrm{HbA}_{1 \mathrm{c}}(\%)$ & $5.39(0.23)$ & $8.78(0.28) * * *$ \\
\hline Beta cell glucose sensitivity ${ }^{a}$ & $187.9(44.8)$ & $16.6(3.3) * * *$ \\
\hline Rate sensitivity ${ }^{b}$ & $758.9(165)$ & $175.3(60.7) *$ \\
\hline Potentiation factor ratio & $1.892(0.44)$ & $1.117(0.111) *$ \\
\hline Basal insulin secretion ${ }^{c}$ & $107.1(10.5)$ & $141.7(13.4) *$ \\
\hline Integral of total insulin secretion OGTT ${ }^{d}$ & $52.7(6.9)$ & $30.9(3.6) * * *$ \\
\hline
\end{tabular}


degree in both groups during the clamp. Both groups were insulin-resistant; however glucose disposal was markedly reduced in the type 2 diabetic subjects $\left(2.15 \pm 0.42 \mathrm{mg} \mathrm{kg}^{-1}\right.$ $\min ^{-1}$ ) compared with obese control subjects $(4.09 \pm$ $\left.0.58 \mathrm{mg} \mathrm{kg}^{-1} \min ^{-1} ; p<0.03\right)$.

OGTT and IVGTT Insulin sensitivity was also calculated from the OGTT, using oral glucose insulin sensitivity (OGIS). The type 2 diabetic subjects were much more insulin-resistant (OGIS $265 \pm 10 \mathrm{ml} \mathrm{min}^{-1} \mathrm{~m}^{-2}$ ) than obese control subjects (OGIS $407 \pm 13 \mathrm{ml} \mathrm{min}^{-1} \mathrm{~m}^{-2} ; p<0.0001$ ). Beta cell function, as assessed by OGTT modelling, was markedly impaired in type 2 diabetic subjects compared with the control group (Table 1). Glucose sensitivity, rate sensitivity and the potentiation factor ratio were all significantly reduced in type 2 diabetic subjects. Glucose sensitivity was strongly correlated with mean glucose levels during the OGTT in the whole group $(r=0.95 p<0.0001$, after log-transformation). Similar results were obtained with 2-h glucose.

In the IVGTT, the type 2 diabetic subjects subjects had markedly reduced beta cell response to glucose. Both acute insulin response $(389 \pm 62$ vs $29 \pm 71 \mathrm{pmol} / \mathrm{l})$ and the equivalent C-peptide measurements $(997 \pm 126$ vs $45 \pm$ $80 \mathrm{pmol} / \mathrm{l}$ ) were reduced in the type 2 diabetic subjects subjects, as well as the incremental area of insulin secreted after the glucose bolus $\left(435 \pm 62 \mathrm{vs} 19 \pm 49 \mathrm{pmol} \mathrm{min}^{-1} \mathrm{~m}^{-2}\right)$.

\section{Effects of exercise intervention}

The exercise intervention was completed by 14 of the obese control subjects and seven of the diabetic subjects.

Clinical and laboratory measurements Table 2 shows that there was no change in body weight, BMI, blood pressure or percent body fat in either group at the end of the 3-month exercise programme. Waist circumference was reduced in the type 2 diabetic subjects $(115.2 \pm 5.5$ vs $110.7 \pm 5.1 \mathrm{~cm}$, $p=0.031$ ), with a similar but not significant trend in control subjects $(100.8 \pm 3.3$ vs $96.7 \pm 2.9 \mathrm{~cm}, p=0.075) . V \mathrm{O}_{2 \max }$ increased more than $20 \%$ in the obese control subjects (from $2.77 \pm 0.24$ to $3.36 \pm 0.41 \mathrm{ml} \mathrm{min}{ }^{-1} \mathrm{~kg}^{-1} p<0.0001$ ), but did not improve significantly in the type 2 diabetic subjects ( $2.48 \pm 0.31$ to $2.72 \pm 0.35 \mathrm{ml} \mathrm{min}{ }^{-1} \mathrm{~kg}^{-1}, p=0.078$ ) (Fig. 1a). Change in $V \mathrm{O}_{2 \max }$ in the obese group was positively correlated with change in glucose disposal $(r=$ $0.55, p=0.05$ ). Mean glucose levels during the OGTT did not change in either group after exercise. There were no significant changes in fasting lipids. Fasting concentrations of NEFA tended to be higher at baseline in the type 2 diabetic subjects, although this did not reach significance. However, after completion of the exercise, NEFA were significantly lower in the control group than in the type 2 diabetic group, in whom exercise induced no change (Fig. 1b).

Hyperinsulinaemic-euglycaemic clamp studies Basal hepatic glucose output was not changed in either group at the end of the exercise intervention (Fig. 1c). Suppression of hepatic glucose output during the clamp remained similar in both groups and was unchanged after the exercise intervention (Fig. 1d). Exercise intervention led to no significant change in whole-body glucose disposal in either group ( $4.31 \pm 0.71$ vs $5.33 \pm 0.7 \mathrm{mg} \mathrm{kg}^{-1} \mathrm{~min}^{-1}$ in the control, $2.5 \pm$ 0.3 vs $2.57 \pm 0.42 \mathrm{mg} \mathrm{kg}^{-1} \mathrm{~min}^{-1}$ in the type 2 diabetic subjects, $p=\mathrm{NS}$ ) (Fig. 1e).

OGTT and IVGTT Insulin sensitivity calculated from OGIS did not improve in either group after exercise intervention ( $417 \pm 14$ vs $420 \pm 16$ in the control group, $p=\mathrm{NS}$; $281 \pm 16$ vs $260 \pm 21$ in the type 2 diabetic group, $p=0.05$ ) (Fig. 1f). All measures of insulin secretion remained unchanged in both groups after exercise.

\section{Discussion}

Marked visceral obesity and extreme insulin resistance are associated with early-onset type 2 diabetes. This form of diabetes is a growing public health problem. While visceral obesity is an important risk factor, the natural history of this condition in younger people is not known. Young patients with type 2 diabetes have well established loss of beta cell insulin secretion at the time of clinical presentation [7]. Two possibilities arise: (1) early-onset type 2 diabetes is identical in pathogenesis and clinical course to type 2 diabetes of later onset; or (2) type 2 diabetes of early onset is a more extreme phenotype with even greater cardiovascular and lifelong risk of complications than in older subjects. We have previously shown that patients in our clinic with onset of diabetes under 40 years are significantly more obese at an earlier age, have worse cardiovascular risk profiles than their older counterparts and have worse initial and ongoing glycaemic control, despite exactly similar care [6]. We have also reported that young type 2 subjects have a markedly adverse cardiovascular risk profile, as already established in a cohort with mean age of 22 years [7]. The clinical and research evidence to date support the hypothesis that early-onset type 2 diabetes is an extreme phenotype, distinct from the phenotype of older subjects with typical type 2 diabetes and from that of young obese subjects with normal glucose tolerance.

In the current study, we conducted baseline studies of whole-body and hepatic glucose turnover using the clamp 
Table 2 Clinical data, lipid profiles and metabolic data derived from the OGTT: comparison between obese non-diabetic control and type 2 diabetes subjects pre and post exercise

\begin{tabular}{|c|c|c|c|c|}
\hline \multirow[b]{2}{*}{ Fixed data } & \multicolumn{2}{|l|}{ Control subjects } & \multicolumn{2}{|c|}{ Type 2 diabetic subjects } \\
\hline & & & & \\
\hline$n$ & \multicolumn{2}{|l|}{14} & \multicolumn{2}{|l|}{7} \\
\hline Male : female ratio & \multicolumn{2}{|l|}{$4: 10$} & \multicolumn{2}{|l|}{$3: 4$} \\
\hline Mean age (years) & \multicolumn{2}{|l|}{$25.6(0.9)$} & \multicolumn{2}{|l|}{$26.1(0.9)$} \\
\hline Per exercise status & Pre-exercise & Post-exercise & Pre-exercise & Post-exercise \\
\hline Weight (kg) & $97.47(4.65)$ & $95.6(4.54)$ & $108.3(8.7)$ & $109.4(8.4)$ \\
\hline BMI $\left(\mathrm{kg} / \mathrm{m}^{2}\right)$ & $34.25(1.44)$ & $33.58(1.34)$ & $35.63(1.23)$ & $36.02(1.17)$ \\
\hline Systolic BP (mmHg) & $116.1(4)$ & $112.8(4)$ & $119(4.7)$ & $121(4.2)$ \\
\hline Diastolic BP (mmHg) & $71.3(2.4)$ & $69.6(2.3)$ & $77(2.5)$ & $81(2.3)$ \\
\hline Waist circumference $(\mathrm{cm})$ & $100.8(3.3)$ & $96.7(2.9)$ & $115.2(5.5)$ & $110.7(5.1) *$ \\
\hline Hip circumference $(\mathrm{cm})$ & $113.3(3.4)$ & $107.1(3) * * *$ & $116.6(4.3)$ & $115.3(3.7)$ \\
\hline WHR & $0.89(0.02)$ & $0.9(0.02)$ & $0.99(0.05)$ & $0.96(0.03)$ \\
\hline Body fat $\%$ & $39(2.4)$ & $38.4(2.3)$ & $39.9(3.1)$ & $39.7(3.1)$ \\
\hline$V \mathrm{O}_{2 \max }\left(\mathrm{ml} \mathrm{min} \operatorname{mg}^{-1} \mathrm{~kg}^{-1}\right)$ & $2.77(0.24)$ & $3.36(0.41) * * *$ & $2.48(0.31)$ & $2.72(0.35)$ \\
\hline Fasting plasma glucose $(\mathrm{mmol} / \mathrm{l})$ & $4.97(0.11)$ & $4.92(0.11)$ & $9.39(1.06)$ & $10.64(1.55)$ \\
\hline Total cholesterol (mmol/l) & $4.47(0.26)$ & $4.25(0.26)$ & $4.47(0.3)$ & $4.52(0.24)$ \\
\hline HDL-cholesterol (mmol/1) & $1.12(0.09)$ & $1.11(0.06)$ & $0.96(0.05)$ & $0.95(0.05)$ \\
\hline LDL-cholesterol (mmol/l) & $2.46(0.22)$ & $2.83(0.25)$ & $2.6(0.26)$ & $2.88(0.18)$ \\
\hline Triacylglycerols $(\mathrm{mmol} / \mathrm{l})$ & $1.37(0.2)$ & $1.19(0.13)$ & $2.63(0.39)$ & $2.26(0.29)$ \\
\hline Fasting plasma NEFA (mmol/l) & $0.635(0.053)$ & $0.561(0.036)$ & $0.820(0.104)$ & $0.826(0.043)$ \\
\hline $\mathrm{HbA}_{1 \mathrm{c}}(\%)$ & $5.43(0.11)$ & $5.32(0.16)$ & $8.46(0.6)$ & $8.41(0.73)$ \\
\hline Beta cell glucose sensitivity ${ }^{a}$ & $145.19(24.68)$ & $128.81(17.39)$ & $13.29(2.92)$ & $21.01(7.44)$ \\
\hline Rate sensitivity (pmol m $\mathrm{m}^{-2} \mathrm{mmol}^{-1} \mathrm{l}^{-1}$ ) & $795.55(195.44)$ & $1095.93(267.62)$ & $241.38(108.95)$ & $196.45(56.87)$ \\
\hline Potentiation factor ratio & $1.44(0.14)$ & $1.57(0.19)$ & $1.29(0.18)$ & $1.1(0.12)$ \\
\hline Basal insulin secretion $\left(\mathrm{pmol} \min ^{-1} \mathrm{~m}^{-2}\right.$ ) & $100.05(12.58)$ & $102.1(9.53)$ & $146.43(15.15)$ & $140.56(12.42)$ \\
\hline Integral of total insulin secretion OGTT ${ }^{b}$ & $43.41(2.12)$ & $44.13(3.08)$ & $29.32(2.61)$ & $34.31(6.48)$ \\
\hline
\end{tabular}

Figures in brackets: SEM

a $\mathrm{pmol} \mathrm{min}^{-1} \mathrm{~m}^{-2} \mathrm{mmol}^{-1} \mathrm{l}^{-1}$

b $\mathrm{nmol} / \mathrm{m}^{2}$

$* p<0.05 ; * * * p<0.001$

technique with deuterated glucose tracer. We used wholebody $V \mathrm{O}_{2 \max }$ as an index of physical fitness. Our subjects were two matched groups of severely obese young subjects, one with type 2 diabetes, the other non-diabetic. The latter non-diabetic group was nonetheless severely insulin-resistant. However, the young type 2 diabetic subjects were even more insulin-resistant at baseline. Whole-body glucose disposal was dramatically reduced and hepatic glucose production was elevated under fasting conditions. During the clamp studies, suppression of hepatic glucose production was similar between the two groups, in contrast to whole-body glucose disposal, which was significantly reduced in the diabetes group. Insulin secretion was markedly reduced in the type 2 diabetic group. The characteristics of beta cell dysfunction in young type 2 diabetic subjects are similar to those we have observed in older diabetic patients [26]. In particular, young type 2 diabetic subjects show the classical marked decrease in glucose sensitivity, rate sensitivity and potentiation factor ratio. In addition, the typical associations between mean or 2-h glucose and both beta cell glucose sensitivity and insulin sensitivity were also observed in this group. However, the association with glucose tolerance was stronger for beta cell glucose sensitivity than for insulin sensitivity, suggesting that in this insulin-resistant population the major cause of glucose intolerance is beta cell dysfunction.

Recent studies from a group in Ohio, USA, used OGTT and IVGTT to show that adolescents with type 2 diabetes have significant insulin resistance, even compared with non-diabetic subjects of similar obesity and body fatness, and impaired insulin secretion relative to their degree of insulin resistance [28]. In contrast to our findings, the diabetic subjects in those studies retained a first-phase insulin response to glucose that was comparable with lean 

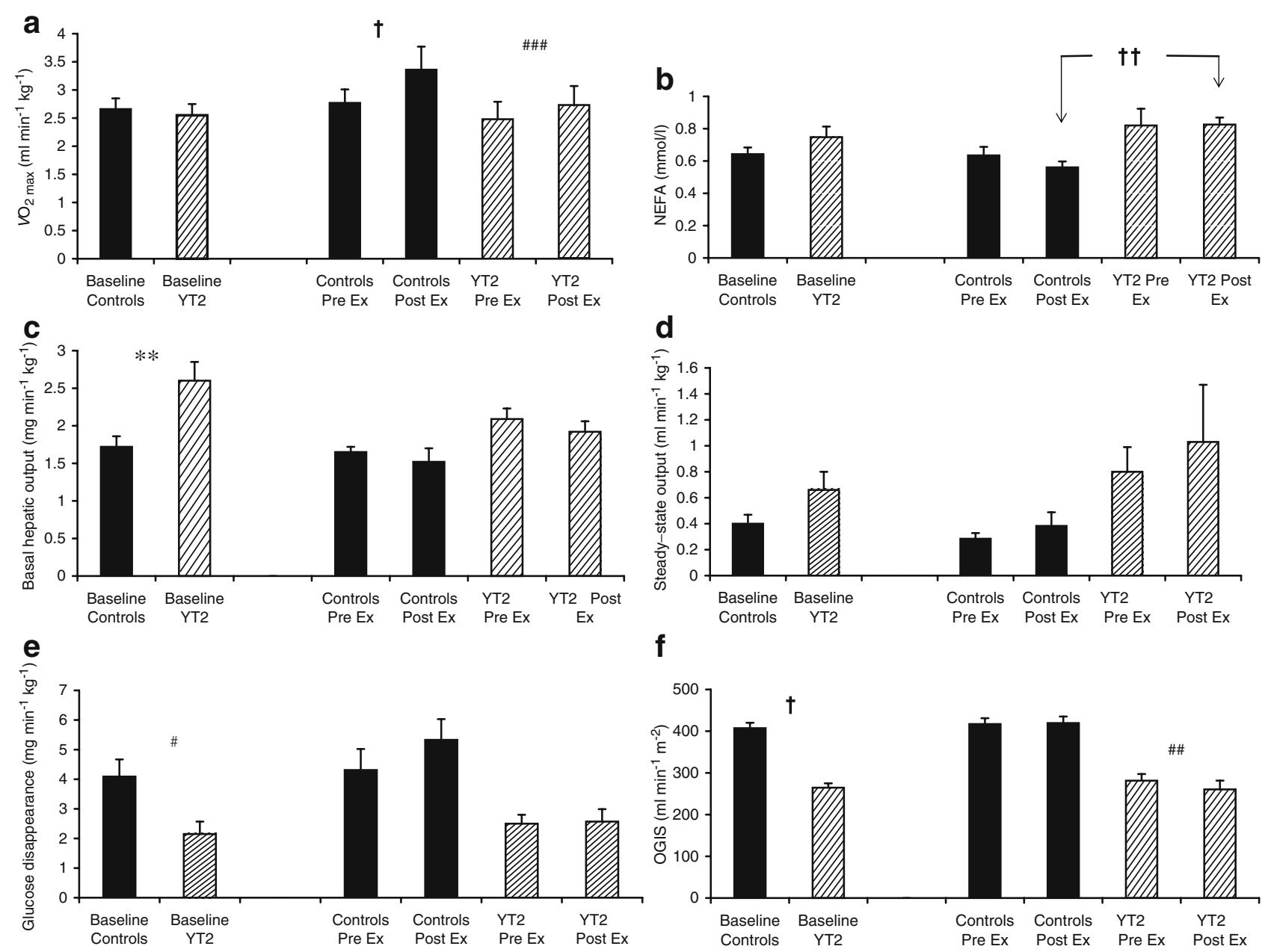

Fig. 1 Comparison of measurements between obese non-diabetic control and type 2 diabetes subjects at baseline and then pre-exercise (Pre Ex) and post-exercise (Post Ex) in those who completed the exercise programme. a $V \mathrm{O}_{2 \max } ;$ b fasting NEFA; c basal hepatic

control subjects. Another group conducted hyperinsulinaemic-euglycaemic clamp studies in six French subjects with type 2 diabetes and a median age of 15.4 years [29]. Similar to our study, they demonstrated marked insulin resistance and beta cell failure although these subjects had very good chronic glycaemic control.

Aerobic exercise has been shown to be effective in improving insulin sensitivity in patients with obesity, prediabetes and type 2 diabetes $[13,14,16]$. The landmark diabetes prevention studies have proven the efficacy of exercise intervention in preventing progression from IGT to diabetes $[9,11,12]$. We have recently shown that just 7 days of exercise training led to increased glucose disposal and muscle GLUT4 protein content in middle-aged, obese subjects with type 2 diabetes (mean age 45 years, BMI $36 \mathrm{~kg} / \mathrm{m}^{2}$ ) [15]. Since early-onset type 2 diabetes is characterised by obesity and severe insulin resistance, we chose to investigate the effect of exercise intervention in glucose output (clamp); d steady-state hepatic glucose output (clamp); e glucose disappearance during clamp; and f OGIS. Error bars: SEM. YT2, young type 2 diabetic subjects. ${ }^{* *} p=0.001,{ }^{\#} p=0.03$; ${ }^{\# \#} p=$ $0.047 ;{ }^{\# \#} p=0.078 ;{ }^{\dagger} p<0.0001 ;{ }^{\dagger \dagger} p=0.0003$

younger subjects with type 2 diabetes. We hypothesised that exercise alone, while maintaining a stable diet, should improve insulin sensitivity. The advantage of the study design was that all exercise sessions took place at our research unit and each session was supervised by either the exercise physiologist or study physician. The intensity of the exercise was constantly scrutinised using continuous heart rate monitoring. We only compared measurements in subjects who actually completed the study (14 control and seven type 2 diabetic subjects). To our surprise, there were essentially no metabolic improvements in either study group at the end of the 3-month programme. However, the obese control group had a $20 \%$ increase in $V \mathrm{O}_{2 \max }$, which was positively correlated with glucose disposal, and a reduction in fasting NEFA when compared with the diabetic group, despite identical compliance with the training programme.

This raises interesting new questions about the pathogenesis and treatment of early-onset type 2 diabetes in 
obese young people. The duration (3 months) of exercise at the frequency and intensity chosen for the current study may simply have been insufficient for the improvements that were expected. However, previous studies including our own in equally obese but older subjects with obesity and type 2 diabetes, have shown improvements in insulin sensitivity and glycaemic control with similar or even less rigorous exercise regimes [13, 14]. Another question is whether it would have been preferable to combine the exercise regime with a weight-losing low-energy diet. However, subjects in the current study were instructed to maintain a stable diet during the 3-month exercise programme, and compliance with this diet regime was satisfactory. We chose to study the effect of exercise alone, not least because it was possible to accurately document exercise compliance, but also because we have found that exercise is an attractive behavioural approach to improving overall lifestyle in this kind of subject group. In addition, an alteration of diet, in conjunction with exercise, would have made it more difficult to interpret any changes in physiological measurements.

In 2005 a group in Canada reported the effects of exercise training on glucose homeostasis in almost 600 subjects, using a standard IVGTT [30]. While they observed a mean increase of $10 \%$ in insulin sensitivity after 20 weeks of aerobic training, between-subject variations in metabolic response to exercise were large, not unlike our study. In fact, most subjects showed a deterioration in the acute insulin response to glucose. It has been suggested that progressive resistance training confers greater metabolic benefits than aerobic training [31, 32]. While the exercise modality may also be relevant, most studies to date, including our own, have examined the effects of aerobic exercise training.

In this previously sedentary cohort it is also possible that our subjects were less active than previously outside the training times. We did not make any specific measurements of overall physical activity in the current study and we think it unlikely that this could have been reduced. While devices are being developed to measure physical activity continuously over several days [33], we did not use these in our study.

More basic physiological and biochemical mechanisms might explain the non-response to exercise in these patients. For example, certain diabetes susceptibility genotypes might predispose individuals to respond or not to respond to exercise. It is possible that subjects with early-onset type 2 diabetes (and severe obesity) have either genetic or acquired factors that confer resistance at the level of skeletal muscle to the expected benefits of aerobic exercise training. It is now widely recognised that mitochondrial dysfunction contributes significantly to insulin resistance and loss of insulin secretion in type 2 diabetes. The failure to increase $V \mathrm{O}_{2 \max }$ in the diabetes subjects is consistent with mitochondrial dysfunction, which could result from a number of causes, including persistent lipotoxicity, which did not improve in this group after exercise. Further studies will be required to address these questions, which are so important for the development of effective treatments for these high-risk patients.

Acknowledgements This study was funded by the Diabetes Education and Research Fund and in part by unrestricted educational grants from Pfizer and Novo Nordisk. The authors are especially grateful to the study participants.

Duality of interest None of the authors have any duality of interest to declare.

\section{References}

1. Wild S, Roglic G, Green A et al (2004) Global prevalence of diabetes: estimates for the year 2000 and projections for 2030 . Diabetes Care 27:1047-1053

2. Bloomgarden ZT (2004) Type 2 diabetes in the young: the evolving epidemic. Diabetes Care 27:998-1010

3. McCarthy SN, Harrington KE, Kiely M et al (2001) Analyses of the anthropometric data from the North/South Ireland Food Consumption Survey. Public Health Nutr 4:1099-1106

4. Lissau I, Overpeck MD, Ruan WJ et al (2004) Body mass index and overweight in adolescents in 13 European countries, Israel, and the United States. Arch Pediatr Adolesc Med 158:27-33

5. No authors listed (2003) The National Health and Lifestyle Surveys. Survey of lifestyle, attitudes and nutrition (SLAN) \& The Irish Health Behaviour in School-Aged Children Survey (HBSC). Health Promotion Unit, Department of Health and Children, Dublin

6. Hatunic M, Burns N, Finucane F et al (2005) Contrasting clinical and cardiovascular risk status between early and later onset type 2 diabetes. Diab Vasc Dis Res 2:73-75

7. McQuaid S, O'Gorman DJ, Yousif O et al (2005) Early-onset insulin-resistant diabetes in obese Caucasians has features of typical type 2 diabetes, but 3 decades earlier. Diabetes Care 28:1216-1218

8. Helmrich SP, Ragland DR, Leung RW, Paffenbarger RS Jr (1991) Physical activity and reduced occurrence of non-insulin-dependent diabetes mellitus. N Engl J Med 325:147-152

9. Knowler WC, Barrett-Connor E, Fowler SE et al (2002) Reduction in the incidence of type 2 diabetes with lifestyle intervention or metformin. N Engl J Med 346:393-403

10. Manson JE, Rimm EB, Stampfer MJ et al (1991) Physical activity and incidence of non-insulin-dependent diabetes mellitus in women. Lancet 338:774-778

11. Pan XR, Li GW, Hu YH et al (1997) Effects of diet and exercise in preventing NIDDM in people with impaired glucose tolerance. The Da Qing IGT and Diabetes Study. Diabetes Care 20:537-544

12. Tuomilehto J, Lindstrom J, Eriksson JG et al (2001) Prevention of type 2 diabetes mellitus by changes in lifestyle among subjects with impaired glucose tolerance. N Engl J Med 344:1343-1350

13. DeFronzo RA, Sherwin RS, Kraemer N (1987) Effect of physical training on insulin action in obesity. Diabetes 36:1379-1385

14. Hughes VA, Fiatarone MA, Fielding RA et al (1993) Exercise increases muscle GLUT-4 levels and insulin action in subjects with impaired glucose tolerance. Am J Physiol 264:E855-E862 
15. O'Gorman DJ, Karlsson HK, McQuaid S et al (2006) Exercise training increases insulin-stimulated glucose disposal and GLUT 4 (SLC2A4) protein content in patients with type 2 diabetes. Diabetologia 49:2983-2992

16. Houmard JA, Tanner CJ, Slentz CA et al (2004) Effect of the volume and intensity of exercise training on insulin sensitivity. J Appl Physiol 96:101-106

17. Burstein R, Polychronakos C, Toews CJ et al (1985) Acute reversal of the enhanced insulin action in trained athletes. Association with insulin receptor changes. Diabetes 34:756-760

18. Dela F, Larsen JJ, Mikines KJ et al (1995) Insulin-stimulated muscle glucose clearance in patients with NIDDM. Effects of onelegged physical training. Diabetes 44:1010-1020

19. Heath GW, Gavin JR 3rd, Hinderliter JM et al (1983) Effects of exercise and lack of exercise on glucose tolerance and insulin sensitivity. J Appl Physiol 55:512-517

20. Schneider SH, Amorosa LF, Khachadurian AK, Ruderman NB (1984) Studies on the mechanism of improved glucose control during regular exercise in type 2 (non-insulin-dependent) diabetes. Diabetologia 26:355-360

21. Ludvik B, Nolan JJ, Baloga J et al (1995) Effect of obesity on insulin resistance in normal subjects and patients with NIDDM. Diabetes 44:1121-1125

22. Nolan JJ, Freidenberg G, Henry R et al (1994) Role of human skeletal muscle insulin receptor kinase in the in vivo insulin resistance of noninsulin-dependent diabetes mellitus and obesity. J Clin Endocrinol Metab 78:471-477

23. Nolan JJ, Ludvik B, Baloga J et al (1997) Mechanisms of the kinetic defect in insulin action in obesity and NIDDM. Diabetes 46:994-1000

24. Mari A, Schmitz O, Gastaldelli A et al (2002) Meal and oral glucose tests for assessment of beta-cell function: modeling analysis in normal subjects. Am J Physiol Endocrinol Metab 283:E1159-E1166

25. Mari A, Tura A, Gastaldelli A, Ferrannini E (2002) Assessing insulin secretion by modeling in multiple-meal tests: role of potentiation. Diabetes 51(Suppl 1):S221-S226

26. Ferrannini E, Gastaldelli A, Miyazaki Y et al (2005) Beta-cell function in subjects spanning the range from normal glucose tolerance to overt diabetes: a new analysis. J Clin Endocrinol Metab 90:493-500

27. Van Cauter E, Mestrez F, Sturis J, Polonsky KS (1992) Estimation of insulin secretion rates from C-peptide levels. Comparison of individual and standard kinetic parameters for C-peptide clearance. Diabetes 41:368-377

28. Elder DA, Prigeon RL, Wadwa RP et al (2006) Beta-cell function, insulin sensitivity, and glucose tolerance in obese diabetic and nondiabetic adolescents and young adults. J Clin Endocrinol Metab 91:185-191

29. Druet C, Tubiana-Rufi N, Chevenne D et al (2006) Characterization of insulin secretion and resistance in type 2 diabetes of adolescents. J Clin Endocrinol Metab 91:401-404

30. Boule NG, Weisnagel SJ, Lakka TA et al (2005) Effects of exercise training on glucose homeostasis: the HERITAGE Family Study. Diabetes Care 28:108-114

31. Cuff DJ, Meneilly GS, Martin A et al (2003) Effective exercise modality to reduce insulin resistance in women with type 2 diabetes. Diabetes Care 26:2977-2982

32. Willey KA, Singh MA (2003) Battling insulin resistance in elderly obese people with type 2 diabetes: bring on the heavy weights. Diabetes Care 26:1580-1588

33. Brage S, Brage N, Franks PW et al (2005) Reliability and validity of the combined heart rate and movement sensor Actiheart. Eur J Clin Nutr 59:561-570 